\title{
The Effects of Heart Rate Versus Speed-Based High- Intensity Interval Training on Heart Rate Variability in Young Females
}

\author{
Maryam Rabbani ${ }^{1}$, Effat Bambaeichi ${ }^{2 *}$, Fahimeh Esfarjani ${ }^{2}$, Alireza Rabbani ${ }^{3}$ \\ ${ }^{1} \mathrm{MSc}$, Department of Exercise Physiology, Faculty of Sport Sciences, University of Isfahan, Isfahan, Iran \\ ${ }^{2}$ Associate Professor, Department of Exercise Physiology, Faculty of Sport Sciences, University of Isfahan, Isfahan, Iran \\ ${ }^{3} \mathrm{PhD}$ Student, Department of Exercise Physiology, Faculty of Sport Sciences, University of Isfahan, Isfahan, Iran
}

\section{*Correspondence to Effat Bambaeichi, Tel: +989132050472 Email: e.bambaeichi@spr.ui.ac.ir}

Received December 4, 2016 Accepted June 17, 2017 Published online June 30, 2017

Please cite this article as follows: Rabbani M, Bambaeichi E, Esfarjani F, Rabbani A. The effects of heart rate versus speed-based high-intensity interval training on heart rate variability in young females. Int J Basic Sci Med. 2017;2(2):9094. doi:10.15171/ ijbms.2017.17.

\begin{abstract}
Introduction: The aim of this study was to compare the effects of high-intensity interval training (HIT) prescription by heart rate (HR-based) and running speed (speed-based) methods on natural logarithm of the square root of the mean of the sum of the squares of differences between adjacent normal R-R intervals (Ln rMSSD) as a measure of heart rate variability (HRV) in young female student athletes.

Methods: Seventeen female student athletes participated in this study and were divided into HR-based ( $n=9$, age: 16.7 years) and speed-based ( $n=8$, age: 16.9 years) HIT groups. 30-15 Intermittent Fitness Test was used for the speed-based group to detect the reference maximum speed $\left(\mathrm{V}_{\mathrm{IFT}}\right)$ for prescribing the HIT intensity accordingly. Age predicted maximal HR was used for the HR-based group as the reference value. All subjects performed similar training protocol for 5 weeks, except the method of individualizing HIT sessions (2 weekly sessions of HIT=3 sets of 3 minutes work interspersed with 3 minutes passive recovery with the 15-15 seconds format during each working set); either according to $90 \%-95 \%$ of maximal $\mathrm{HR}$ or $\mathrm{V}_{\mathrm{IFT}}$.

Results: HR- and speed-based HIT groups showed the most likely large improvements in Ln rMSSD of $+7.9 \%$, 90\% confidence limits [CL] $(5.9 ; 10.0)$; standardized change: $+1.75(1.32 ; 2.19)$ and $+5.5 \%$, $(2.8 ; 8.3) ;+1.41(0.72 ; 2.09)$, respectively. In between group analyses, HR-based HIT produced likely a small greater improvement in Ln rMSSD than speed-based HIT (+1.9\%, [-5.0; $4.4] ;+0.50$ [-0.14; 1.14], chances for greater/similar/lower values of 79/17/4).

Conclusion: It is concluded that both HIT prescription strategies were effective in Ln rMSSD elevation, but using maximal HR as a reference may elicit higher parasympathetic dominance with small effect in young female student athletes.

Keywords: Heart Rate, High-intensity interval training, Fitness, Heart rate variability, Females
\end{abstract}

improve HRV profile in both patient and athletes' populations by enhancing parasympathetic dominance. ${ }^{4,6-9}$

When individualizing HIT programs, different variables including the training intensity need to be taken into account. ${ }^{10,11}$ Accordingly, the use of maximal heart rate $\left(\mathrm{HR}_{\max }\right)$ or the final speed reached in 30-15 Intermittent Fitness Test $\left(30-15_{\text {IFT }}, \mathrm{V}_{\text {IFT }}\right.$ ) as a reference, are two approaches known as HR-based and speed-based methods, respectively. ${ }^{10}$ Although HR-based method has several proposed limitations including difficulties for practitioners when controlling exercise intensity, it is yet the most

Copyright $(2017$ The Author(s); Published by Zabol University of Medical Sciences. This is an open-access article distributed under the terms of the Creative Commons Attribution License (http://creativecommons.org/licenses/by/4.0), which permits unrestricted use, distribution, and reproduction in any medium, provided the original work is properly cited. 
common method implemented in the field. ${ }^{10}$

Furthermore, there are a number of proposed theoretical superiorities of speed-based HIT by using $\mathrm{V}_{\text {IFT }}$ as a reference over the HR-based methods including its higher sensitivity to the athlete's locomotor profile, acceleration, deceleration, and change of direction abilities. ${ }^{12}$ It has also been shown that $\mathrm{V}_{\mathrm{IFT}}$-based method is more effective than HR-based HIT to improve maximal high-intensity running performance among the athletes. ${ }^{13}$ However, differences between these two approaches (i.e., speed-based vs. HR-based) in improving the physiological adaptations like HRV have not been investigated yet.

In addition, while analyzing HRV includes a myriad of variables in three different aspects (i.e., time domain, frequency domain and spectral analyses), the use of the square root of the mean of the sum of the squares of differences between adjacent normal R-R intervals ( $\mathrm{Ln}$ rMSSD) has been recommended as a valid and sensitive marker for monitoring training-induced physiological adaptations in athletes. ${ }^{14}$ Fortunately, advancements in smart phone apps have recently provided ultra-shorttime Ln rMSSD as a valid index. ${ }^{15}$ However, while it has been shown that ultra-short-time Ln rMSSD, is sensitive to team sport training interventions, ${ }^{16}$ the differences between the effects of different HIT protocols on this marker have yet to be determined.

Following these lines, there seems to be a lack of evidence on differences between the effects of HR-based and speed-based HIT interventions on ultra-short-time Ln rMSSD in the literature. Therefore, the purpose of this study was to investigate the within group changes and between group differences in changes of ultra-short-time Ln rMSSD following 5 weeks of speed-based and Heartbased HIT interventions in young female student athletes.

\section{Methods}

Subjects

Seventeen female student athletes participated in this quasi-experimental study and were divided into HRbased $(\mathrm{n}=9$, mean $\pm \mathrm{SD}$; age: $16.7 \pm 0.3$ years, weight: $59.8 \pm 2.6 \mathrm{~kg}$, height: $162.4 \pm 2.7 \mathrm{~cm}$ ) and speed-based $(\mathrm{n}=8$, age: $16.9 \pm 0.3$ years, weight: $58.7 \pm 3.2 \mathrm{~kg}$, height: $164.3 \pm 3.3 \mathrm{~cm}$ ) HIT groups. Before the study, pre levels of resting HRV were used to allocate the participants in 2 homogenous experimental groups. First explanations about the experimental risks of the study were given and later informed consent was obtained from all the participants and their parents. Furthermore, local research eth- ics committee approved the protocol and the study conformed to the Declaration of Helsinki. ${ }^{17}$

\section{Testing and Training}

The speed-based group performed $30-15_{\text {IFT }}^{12}$ before HIT intervention. $30-15_{\mathrm{IFT}}$ is an incremental high-intensity intermittent running performance field test. The final speed derived from this test (i.e., $\mathrm{V}_{\mathrm{IFT}}$ ) is a reference to individualize speed-based HITs. ${ }^{18} 30-15_{\text {IFT }}$ was performed at the same time of day (10 AM) with similar temperature ranging between $26^{\circ} \mathrm{C}$ to $28^{\circ} \mathrm{C}$. The intensity in both groups was individualized to train the subjects in their red zone (i.e., $>90 \% \mathrm{HR}_{\max }$ ). Age predicted $\mathrm{HR}_{\max }$ was used for the HR-based group as the reference value. ${ }^{19}$ Participants in the HR-based group had to check the Polar Beat ${ }^{\mathrm{TM}}$ application installed on their smart-phone regularly during HIT sessions while using a HR Bluetooth sensor (Polar H7, Finland) to assure exercising in the prescribed zone. The individualized running pace using $\mathrm{V}_{\mathrm{IFT}}$ has been calculated for each subject in the speed-based group. All $\mathrm{V}_{\mathrm{I}}$ ${ }_{\mathrm{FT}}$-based subjects were guided to set their running pace according to an audio signal. While it was not mandatory for the HR-based group to set their running speed according to auditory signal, the running speed for the $\mathrm{V}_{\text {IFT }}$-based group was adjusted according to maximal test speed explained in detail previously. ${ }^{13}$ The experimental period lasted for 5 weeks with 2 weekly HIT sessions. The training protocol during the entire experimental period is shown in Table 1.

\section{Heart Rate Variability Recording}

Home based resting ultra-short-term HRV recording was asked from all subjects in both groups. ${ }^{16}$ The subjects were asked to collect HRV using HRV application installed on their smart phones using HR Bluetooth sensor (H7, Polar, Finland) around fastened their trunk near to xiphoid area as well according to the previous investigations guidelines. ${ }^{16}$ The subjects were guided to record their HRV in the morning before eating and in the supine position. The R-R intervals derived from Elite HRV ${ }^{\mathrm{TM}}$ smart phone application were exported to be analyzed later by Kubios HRV software to calculate Ln rMSSD. ${ }^{20}$ Weekly averages of Ln rMSSD for the first and last week of training intervention were used as pre and post values for statistical analyses.

\section{Statistical Analyses}

Data in the figures are shown as means with $90 \%$ of CI in

Table 1. Training Program

\begin{tabular}{llll}
\hline Week & Protocol & HR-Based Intensity & $\mathrm{V}_{\mathrm{IFT}}$-Based Intensity \\
\hline 1 & 3 sets (6 reps of 15"-15" HIT) & $85-90 \%$ HR max & $90 \% \mathrm{~V}_{\mathrm{IFT}}$ \\
2 & 3 sets (6 reps of 15"-15" HIT) & $85-90 \%$ HR max & $90 \% \mathrm{~V}_{\mathrm{IFT}}$ \\
3 & 3 sets (8 reps of 15"-15" HIT) & $90-95 \%$ HR max & $95 \% \mathrm{~V}_{\mathrm{IFT}}$ \\
4 & 3 sets (8 reps of 15"-15" HIT) & $90-95 \%$ HR max & $95 \% \mathrm{~V}_{\mathrm{IFT}}$ \\
5 & 4 sets (6 reps of 15"-15" HIT) & $90-95 \%$ HR max & $95 \% \mathrm{~V}_{\text {IFT }}$ \\
\hline
\end{tabular}

Note: In all HIT (high-intensity interval training) sessions, work periods were interspersed with 3 min of passive recovery. 
the case. All data were first log-transformed to reduce bias arising from non-uniformity error. Within-group changes and between-groups differences in changes of Ln rMSSD were analyzed. The percentage changes and standardized differences or effect size (ES) with $90 \%$ confidence limits (CL) were used to express the results. ${ }^{21}$ The Hopkins scale was used for standardized change/difference interpretation: < 0.2: Trivial; 0.2 - 0.6: Small; 0.6 - 1.2: Moderate; $>$ 1.2: Large. Magnitude-based inference approach was used to analyze the chance that the true changes were clear or trivial. ${ }^{22}$ Probabilities were also calculated to establish whether the true changes/differences were lower than, similar to, or higher than the smallest worthwhile changes/differences (SWC, $0.2 \times$ between-subjects SD). ${ }^{21}$

\section{Results}

Weekly average of Ln rMSSD values are shown in Table 2. Within-group analyses showed that subjects in the HR-based and $\mathrm{V}_{\mathrm{IFT}}$-based groups had the most likely large improvements in Ln rMSSD of $+7.9 \%$, 90\% CL (5.9; $10.0)$; standardized change: $+1.75(1.32 ; 2.19)$ and $+5.5 \%$, $(2.8 ; 8.3) ;+1.41(0.72 ; 2.09)$, respectively (Figure1A). In between group analyses, HR-based HIT produced likely a small greater improvement in Ln rMSSD than speedbased HIT $(+1.9 \%, 90 \%$ CL $[-5.0 ; 4.4 \%]$; standardized difference: $+0.50[-0.14 ; 1.14]$, chances for greater/similar/ lower values of 79/17/4) (Figue1B).

\section{Discussion}

The aim of the present study was to quantify and compare the respective effects of two methods of individualizing HIT on HRV (i.e., using percentages of either $\mathrm{HR}_{\max }$ or the maximum speed reached during the $30-15_{\text {IFT }}\left(V_{\text {IFT }}\right)$ as a reference value) for the first time. The main result is that while both methods allowed for substantial gains in HRV after 5 weeks, the HR-based approach produced likely a small greater improvement in Ln rMSSD compared with the speed-based approach. These results show that using the HR for individualizing HIT in young female students might be a more efficient choice to develop HRV.

Increases of Ln rMSSD suggest the increased vagal tone activity, parasympathetic dominance and in general the HRV improvement. ${ }^{9,14}$ Although the mechanisms responsible for vagal tone increase are not yet cleared, the angiotensin II and nitric oxide (NO) are potential mediators. Angiotensin II is known as an inhibitor of cardiac vagal tone. ${ }^{23}$ The suppression of angiotensin II expression by training has been addressed by Buch et al. ${ }^{24}$ Documented low levels of angiotensin II inferred from lower levels of plasma renin, ${ }^{25}$ has been referred to be responsible, at least in part, for the higher cardiac vagal tone in athletes. Therefore, there is a possibility of increased cardiac vagal tone activity due to the training-induced suppression of angiotensin II. ${ }^{24}$ The result of our study, confirming the beneficial effect of HIT interventions on Ln rMSSD, is in line with previous investigations reporting the positive outcomes ${ }^{4,6-8}$ and in contrast with studies showing unchanged values in $\mathrm{HRV}^{26,27}$ Discrepancies between
Table 2. Weekly Average of Heart Rate Variability

\begin{tabular}{lcc}
\hline Groups & Pre-test & Post-test \\
\hline HR-based HIT & $3.15 \pm 0.13$ & $3.40 \pm 0.12$ \\
$\mathrm{~V}_{\mathrm{IFT}}$-based HIT & $3.17 \pm 0.11$ & $3.34 \pm 0.09$ \\
\hline
\end{tabular}

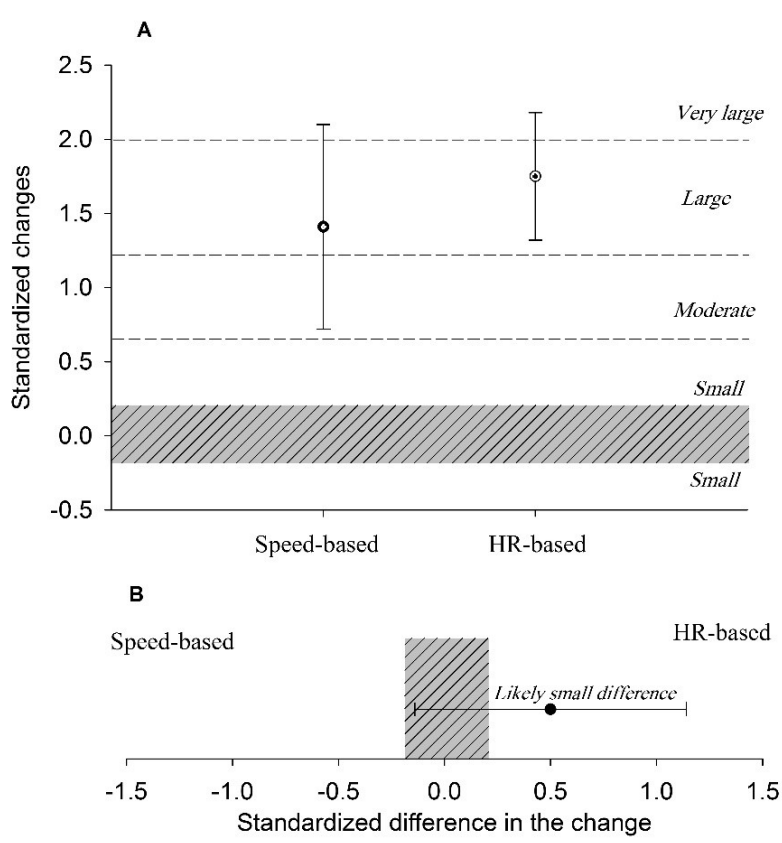

Figure 1. Training-Induced Standardized Changes $(90 \% \mathrm{Cl})$ in Heart Rate Variability of Speed-Based and HR-Based Groups (A) and Standardized Differences Between Changes (90\% CI) (B).

the present study result and previous unchanged HRV reports might be due to the subjects' characteristics ${ }^{27}$ or health conditions. ${ }^{26}$ In fact, in the studies of Currie et $\mathrm{al}^{26}$ and Gamelin et $\mathrm{al}^{27}$ the subjects had coronary artery disease and were prepubescent children, respectively. While the subjects undergoing HIT intervention in the present study were healthy young females.

The interesting finding of the present study when analyzing between group differences in changes was the superiority of HR-based method in improving Ln rMSSD which showed likely a small effect. In fact, $V_{\text {IFT }}$ has been shown to be related not only to the maximal oxygen uptake $\left(\mathrm{VO}_{2 \max }\right)$, but also to the athlete's locomotor profile (i.e., maximal sprinting speed, anaerobic speed reserve, change of direction, acceleration and deceleration). ${ }^{12}$ It seems that HR-based approach targets mainly $\mathrm{VO}_{2 \max }$ and accordingly elicits higher cardiac related physiological adaptations including HRV. ${ }^{28}$

Although speed-based HIT method is an easier method of controlling the intensity in practice, it may have lower longitudinal practicality for over loading the athlete. In fact, HR-based approach ensures that red zone $(>90 \%$ of maximal HR) is always targeted during any HIT session. However, when using speed-based approach, there is not clear perspective on how athlete has been improved in terms of general fitness or $\mathrm{V}_{\mathrm{IFT}}{ }^{29}$ Therefore, there was not any assurance of accurate over loading the athletes and 
training them in their red zones in the last weeks of the plan which may explain less improvement of a physiological marker like HRV in speed-based group.

There is just one similar study in which the effects of these 2 HIT interventions on performance improvement have been compared and the superiority of speed-based approach has been suggested which is different from our study result showing the more effectiveness of HRbased method on HRV. ${ }^{13}$ However, Rabbani and Buchheit ${ }^{13}$ used performance marker (high-intensity running performance) as the training outcome different from our study in which, physiological marker (HRV) was used. Moreover, the limitation of not training both experimental groups in similar time frame in the previous study may further explain such discrepancies. ${ }^{13}$

\section{Conclusion}

This study showed that both speed- and HR-based HIT methods can enhance HRV after 5 weeks in young female student athletes. However, a small greater cardiac physiological adaptation may be elicited when using HR-based approach due to its specificity.

\section{Ethical Approval}

Local ethical committee of Sport Sciences College in the University of Isfahan approved the study protocol as a master proposal in 2016.

\section{Competing Interests}

Authors declare that they have no potential conflict of interest.

\section{Acknowledgments}

The authors thank all participants for their meaningful contributions during the study.

\section{References}

1. Acharya UR, Joseph KP, Kannathal N, Lim CM, Suri JS. Heart rate variability: a review. Med Biol Eng Comput. 2006;44(12):1031-51. doi:10.1007/s11517-006-0119-0.

2. Green KT, Dennis PA, Neal LC, et al. Exploring the relationship between posttraumatic stress disorder symptoms and momentary heart rate variability. J Psychosom Res. 2016;82(31):31-4. doi:10.1016/j. jpsychores.2016.01.003.

3. Vinik AI, Ziegler D. Diabetic cardiovascular autonomic neuropathy. Circulation. 2007;115(3):387-97. doi:10.1161/ CIRCULATIONAHA.106.634949.

4. GuiraudT, Labrunee M, Gaucher-Cazalis K, et al. Highintensity interval exercise improves vagal tone and decreases arrhythmias in chronic heart failure. Med Sci Sports Exerc. 2013;45(10):1861-7. doi:10.1249/ MSS.0b013e3182967559.

5. Achten J, Jeukendrup AE. Heart rate monitoring. Sports Med. 2003;33(7):517-38. doi:10.2165/00007256200333070-00004.

6. Gamelin F, Berthoin S, Sayah H, Libersa C, Bosquet L. Effect of training and detraining on heart rate variability in healthy young men. Int J Sports Med. 2007;28(07):564-70. doi:10.1055/s-2007-964861.
7. Kiviniemi AM, Tulppo MP, Eskelinen JJ, et al. Cardiac autonomic function and high-intensity interval training in middle-age men. Med Sci Sports Exerc. 2014;46(10):19607. doi:10.1249/MSS.0000000000000307.

8. Rennie KL, Hemingway $H$, Kumari M, Brunner E, Malik M, Marmot M. Effects of moderate and vigorous physical activity on heart rate variability in a British study of civil servants. Am J Epidemiol. 2003;158(2):135-43. doi:10.1093/aje/kwg120.

9. Aubert AE, Seps B, Beckers F. Heart rate variability in athletes. Sports Med. 2003;33(12):889-919.

10. Buchheit M, Laursen PB. High-intensity interval training, solutions to the programming puzzle. Sports Med. 2013;43(5):313-38. doi:10.1007/s40279-013-0029-x.

11. Ghalavand A, Motamedi P, Deleramnasab M, Khodadoust M. The Effect of interval training and Nettle supplement on glycemic control and blood pressure in men with type 2 diabetes. Int J Basic Sci Med. 2017(1):33-40. doi:10.15171/ ijbms.2017.08.

12. Buchheit M. The 30-15 intermittent fitness test: accuracy for individualizing interval training of young intermittent sport players. J Strength Cond Res. 2008;22(2):365-74. doi:10.1519/JSC.0b013e3181635b2e.

13. Rabbani A, Buchheit M. Heart rate-based versus speedbased high-intensity interval training in young soccer players. Paper presented at: $4^{\text {th }}$ World Congress on Science and Football VII; October 29 2014; Portland, USA. https:// wordpress.up.edu/wcss2014usa/html. Accessed January $15,2015$.

14. Buchheit M. Monitoring training status with HR measures: do all roads lead to Rome? Front Physiol. 2014;5:7. doi:10.3389/fphys.2014.00073.

15. Flatt AA, Esco MR. Validity of the ithlete ${ }^{\mathrm{TM}}$ Smart phone application for determining ultra-short-term heart rate variability. J Hum Kinet. 2013;1(39):85-92. doi:10.2478/ hukin-2013-0071.

16. Nakamura FY, Flatt AA, Pereira LA, Ramirez-Campillo R, Loturco I, Esco MR. Ultra-short-term heart rate variability is sensitive to training effects in team sports players. J Sports Sci Med. 2015;14(3):602. doi:10.1001/JSS.203.1142.

17. World Medical Association. World Medical Association Declaration of Helsinki: ethical principles for medical research involving human subjects. JAMA. 2013;310(20):2191. doi:10.1001/jama.2013.281053.

18. Buchheit M. Individualizing high-intensityinterval training in intermittent sport athletes with the 30-15 Intermittent Fitness Test. NSCA Hot Topic Series website. http://www.nsca-lift.org/. Published 2011.

19. Tanaka H, Monahan KD, Seals DR. Age-predicted maximal heart rate revisited. J Am Coll Cardiol. 2001;37(1):153-6.

20. Tarvainen MP, Niskanen J-P, Lipponen JA, Ranta-Aho PO, Karjalainen PA. Kubios HRV-heart rate variability analysis software. Comput Methods Programs Biomed. 2014;113(1):210-20. doi:10.1016/j.cmpb.2013.07.024.

21. Hopkins W, Marshall S, Batterham A, Hanin J. Progressive statistics for studies in sports medicine and exercise science. Med Sci Sports Exerc. 2009;41(1):3. doi:10.1249/ MSS.0b013e31818cb278.

22. Batterham AM, Hopkins WG. Making meaningful inferences about magnitudes. Int J Sports Physiol Perform 2006;1(1):50-57. doi:10.1123/ijspp.1.1.50.

23. Townend JN, Al-Ani M, West JN, Littler WA, Coote JH. Modulation of cardiac autonomic control in humans by angiotensin II. Hypertension. 1995;25(6):1270-5. 
doi:10.1161/01.HYP.25.6.1270.

24. Buch AN, Coote JH, Townend JN. Mortality, cardiac vagal control and physical training-what's the link? Exp Physiol. 2002;87(4):423-35. doi:10.1111/j.1469-445X.2002. tb00055.x.

25. Fagard R, Grauwels R, Groeseneken D, et al. Plasma levels of renin, angiotensin II, and 6-ketoprostaglandin F1 alpha in endurance athletes. J Appl Physiol. 1985;59(3):947-52. doi:10.1152/japplphysiol.01051.

26. Currie KD, Rosen LM, Millar PJ, McKelvie RS, MacDonald MJ. Heart rate recovery and heart rate variability are unchanged in patients with coronary artery disease following 12 weeks of high-intensity interval and moderateintensity endurance exercise training. Appl Physiol Nutr
Metab. 2013;38(6):644-50. doi:10.1139/apnm-2012-0354.

27. Gamelin F-X, Baquet G, Berthoin S, et al. Effect of high intensity intermittent training on heart rate variability in prepubescent children. Eur J Appl Physiol. 2009; 105(5):731-8. doi:10.1007/s00421-008-0955-8.

28. Franklin BA, Hodgson J, Buskirk ER. Relationship between percent maximal $\mathrm{O} 2$ uptake and percent maximal heart rate in women. Res Q Exerc Sport. 1980;51(4):616-26. doi: 10.1080/02701367.1980.10609322.

29. Mann TN, Lamberts RP, Lambert MI. High responders and low responders: factors associated with individual variation in response to standardized training. Sports Med. 2014;44(8):1113-24. doi:10.1007/s40279-014-0197-3. 